FORMATION Formation emploi

Revue française de sciences sociales

100 | octobre-décembre 2007

De la formation professionnelle en Suisse

\title{
À quoi ne se réfèrent pas les référentiels métier des agents de développement local?
}

What do occupational standards for local development workers not refer to?

Was berücksichtigen die systematischen Berufsverzeichnisse bei lokalen

Entwicklungshelfern nicht?

\section{Gilles Jeannot}

\section{(2) OpenEdition}

Journals

\section{Édition électronique}

URL : http://journals.openedition.org/formationemploi/1288

DOI : 10.4000/formationemploi.1288

ISSN : 2107-0946

\section{Éditeur}

La Documentation française

\section{Édition imprimée}

Date de publication : 1 octobre 2007

Pagination : 121-135

ISSN : 0759-6340

\section{Référence électronique}

Gilles Jeannot, « À quoi ne se réfèrent pas les référentiels métier des agents de développement local ? », Formation emploi [En ligne], 100 | octobre-décembre 2007, mis en ligne le 01 octobre 2009, consulté le 30 octobre 2020. URL : http://journals.openedition.org/formationemploi/1288 ; DOI : https://doi.org/10.4000/formationemploi.1288 


\title{
Profession
}

À quoi ne se réfèrent pas

les référentiels métier des agents de développement local?

\author{
Par Gilles Jeannot*
}

\begin{abstract}
Confronter les référentiels métier avec les pratiques et les parcours des agents de développement en milieu rural permet de mettre en évidence de nombreux décalages qui proviennent de trois sources.
\end{abstract}

De nouveaux domaines de l'action publique (développement rural et urbain, médiation, politique d'accompagnement vers l'emploi, ...) ont été investis de manière exploratoire par une offre de moyens humains plutôt que par la définition d'un programme précis d'action: des individus sont ainsi recrutés pour résoudre des problèmes avant même qu'un programme précis des tâches n'ait été défini ${ }^{1}$. Le cas des agents de développement en milieu rural en fournit un exemple significatif, qui sera abordé ici depuis les origines, dans les années 70, à nos jours. Les premiers agents de développement ont en effet transformé les anciens modes d'intervention de l'aménagement rural pour inventer les catégories d'action du développement et de l'intercommunalité, avant de les diffuser dans les établissements publics

\footnotetext{
${ }^{1}$ Une première présentation de cet article a été faite le 23 juin 2006 dans le cadre du séminaire « compétences » du Latts/ université Marne-la-Vallée. Je remercie tout particulièrement ses animateurs, Pascal Ughetto et Marie-Christine Combes, pour leurs commentaires éclairants.
}

de coopération intercommunale et les pays ${ }^{2}$, créés en milieu rural après 2000 (Jeannot, 2005). Dans tous

* Gilles Jeannor est chercheur au Laboratoire Techniques Territoires et Sociétés à l'École des ponts et chaussées, université Paris Est. Ses principaux thèmes de recherche sont: Les services publics et les usagers ; La place du travail dans I'action publique ; Les nouvelles formes de "métiers flous " dans l'action publique : chefs de projet, agents de développement, médiateurs ; Le management public et la gestion du personnel dans les administrations. II a publié récemment sur le thème de l'article proposé : Les métiers flous, travail et action publique, Octarès, 2005. "Les métiers flous du développement local », Sociologie du travail, 47, 1, 2005, p. 17-35. 
les cas, le constat est identique. Dans un premier temps, les agents, ainsi directement confrontés à des problèmes à résoudre, ont fait preuve de capacités d'adaptation, ont repéré des espaces d'action, inventé des solutions, souvent reconnues pertinentes par les autorités publiques qui les ont mobilisées. Puis on a assisté à des formes d'institutionnalisation de l'action. L'objectif, partagé par les promoteurs de ce nouveau type d'intervention et par les agents concernés, étant alors de pérenniser ce mode d'action en maintenant son originalité et en garantissant une protection des individus.

Cette quête de pérennisation s'est incarnée dans la recherche d'une « professionnalisation ». L'emploi du terme "professionnalisation » dans un tel contexte recouvre deux approches. La première, orientée vers l'action publique, est de renforcer et généraliser ce qui a été inventé de façon désordonnée en stabilisant des manières de faire des agents concernés (Brévan, Picard, 2000 ; Labruyère, 1999). Dans certains cas, il s'agit de sortir d'une pratique de militantisme dans laquelle l'efficacité est plus liée à un engagement individuel qu'à une pratique professionnelle; dans d'autres cas, d'établir une coupure plus nette entre des savoirs domestiques et des savoirs professionnels. La seconde approche vise à garantir la protection des individus par le contrôle de l'accès aux emplois, phénomène bien connu de la sociologie des professions (Dubar et Tripier, 1998); les associations de professionnels prétendant en effet à «l'insubstituabilité » (Paradeise, 1986) de membres reconnus de la profession pour certaines fonctions. La production d'un référentiel du « métier » apparaît alors comme le point de passage obligé de cette démarche de «professionnalisation».

Dans le cas des agents de développement rural, contrairement à celui des emplois-jeunes affectés à des fonctions de médiation (Demazière, 2004), l'initiative de la professionnalisation est venue, dans la seconde approche, des agents les plus anciens organisés en associations et qui obtiennent un soutien de certaines administrations. Ces agents ont pour beaucoup connu des positions très indépendantes, soit dans des bureaux d'études, soit dans des organismes d'intervention para-administratifs. Ils y ont expérimenté des conditions d'autonomie et la capacité de contacts directs avec la population, qu'ils associent volontiers à la part d'indépendance du professionnel. Ces conditions correspondent moins à la situation des nouveaux arrivés, que les anciens cherchent à enrôler dans ce projet de professionnalisation et qui sont recrutés, pour beaucoup, dans les intercommunalités émergentes. Dès lors, dans quelle mesure les propositions de réformes qui mobilisent le modèle de la profession apportent-elles les réponses les plus adaptées aux deux objectifs de préservation de la spécificité du développement et de protection des individus?

La présente lecture repose sur une analyse critique de l'outil central mobilisé ces dernières années par ces organisations professionnelles, - la production de « référentiels métier » - et plus précisément sur la mise en évidence des décalages entre cet outil et la réalité des pratiques et des parcours professionnels de ces agents. Comme tout outil d'analyse du travail (Dadoy M. et alii, 1990) ces référentiels imposent en effet une certaine réduction de la pratique : «Analyser le travail, c'est d'abord décider de ne retenir qu'une ou quelques-unes des diverses dimensions qui le constituent. Or dans ce domaine comme dans bien d'autres, les résultats sont inscrits dans la méthodologie elle-même et en partie déterminés par les questions d'origine. » (Dugué et Malglaive, 1990, p. 272) En premier lieu, comme le soulignent Oiry et Sulzer (2005), la difficulté nait des points de vues différents sur la même position professionnelle et du rapport de force établi entre ceux-ci. En second lieu, les référentiels, comme outil de gestion des ressources humaines, tendent, comme le souligne MarieChristine Combes (2002), à faire reposer sur l'individu une performance qui doit beaucoup aussi à l'organisation. En troisième lieu, tout référentiel suppose un minimum de décontextualisation. Ceci s'explique notamment par des considérations cognitives : plus la maille est large, plus les circonstances spécifiques d'exercice du métier par tel ou tel agent devront laisser place à des définitions abstraites et générales des fonctions, ce qui pourrait notamment éclairer le choix de ne pas mettre l'accent sur les caractéristiques spécifiques du travail dans une collectivité territoriale au contact d'un élu. La décontextualisation peut aussi s'expliquer par des visées performatives, la production de référentiels visant à produire des effets en fixant des points de repère 
détachés des contextes. Mais, en outre, dans le cas étudié, un certain nombre de décalages relèvent de la projection, par une partie des plus anciens, d'un modèle de profession sur une situation de l'emploi dans ce domaine qui ne relève que partiellement de ce modèle. On s'attachera alors à rapporter à ces différentes causes les décalages observés.

Le relevé des décalages entre référentiels et pratique s'appuiera sur la confrontation entre deux sources. En premier lieu, un corpus de huit référentiels ( $c f$. encadré 1), collectés par la «plateforme de métiers», réseau qui regroupe la plupart des associations professionnelles d'agents de développement rural et les organismes de tutelle, dont la Délégation interministérielle à l'Aménagement et à la Compétitivité des territoires. En second lieu, les résultats d'une enquête portant sur quatre vingt quinze agents de développement ayant travaillé en Franche-Comté entre 1970 et 2003 (enquête quasi exhaustive sur ce territoire). Cette der- nière enquête a permis de collecter des éléments sur les pratiques professionnelles par la voie d'entretiens semi-directifs ; ceux-ci visent à produire une verbalisation de l'activité autour du commentaire de l'agenda et des éléments sur les parcours par la reconstitution des biographies professionnelles ( $c f$. encadré 2).

Après avoir rappelé les liens entre l'évolution du contexte institutionnel du développement rural et les positions des agents de développement, et souligné la convergence des référentiels du corpus étudié, l'article s'attachera à établir le décalage entre ces référentiels et la pratique sur le travail et les compétences et sur la mobilité. Il s'agira en fait de dire à quoi ne se réfèrent pas les référentiels métier des agents de développement. Nous conclurons sur l'effet contre-productif du modèle de la profession et de son outil, le référentiel métier, pour les fins visées de maintien de l'originalité de l'intervention et de protection des individus.

\section{Encadré 1 \\ Corpus des référentiels}

ARADEL, "Les métiers du développeur économique », Les cahiers du développeur économique, $n^{\circ} 2$, mai 1999.

Chaire Unesco sur la formation des professionnels du développement durable, Référentiel des compétences et des métiers dans le développement et la solidarité, compte-rendu de l'atelier de travail, maison internationale, Bordeaux, 18-19 octobre 2001, 22 p.

Association nationale " Agents de développement, un métier à défendre », Référentiel métier : agents de développement local, mars 2001, 15 p.

AFIP, Culture et promotion AEIS, FNCIVAM, FNFR, inter AFOCG, PEC, UNADEL, ARISTEE ou les professionnels du secteur associatif au service du développement rural, 72 p, 1999

CUCES université de Nancy, CNFPT Lorraine, Tenette P. et Vautrin G., Quels référentiels pour les métiers de développement?, janvier 1993, 26 p.

CUCES université de Nancy, Délégation interministérielle à la Ville, Blanc M. et alii, Référentiel de compétences des métiers du développement social urbain : le métier de chef de projet de la politique de la ville, mars 2002, 53 p.

Parcs naturels régionaux de France, Les métiers des parcs naturels régionaux en ligne : www.espacesnaturels.fr/métiers.

Plate-forme "Métiers du développement territorial », Référentiel de compétences, cœur des métiers, septembre 2005, 30 p. 


\section{Encadré 2 \\ Une enquête en Franche-Comté}

L'enquête a été réalisée en 2003 pour le compte du Centre national de la fonction publique territoriale et du ministère de la Recherche $(\mathrm{ACl}$ - action concertée incitative "travail »). Elle ciblait de manière exhaustive les personnes ayant travaillé comme agent de développement en Franche-Comté depuis le début de cette politique en 1970. 95 entretiens avec des agents de développement ont été menés dont 70 en face-à-face.

II n'existe pas de base permettant de couvrir les différents types d'agents de développement ; l'enquête s'appuie donc sur une reconstitution - en suivant à la fois les réseaux d'interconnaissance et les employeurs potentiels - de la liste des "agents de développement» dans une région particulière, la région Franche-Comté, avec la définition suivante de l'agent de développement : pour la période avant 2000, un agent affecté à un territoire et en responsabilité de la mise en œuvre des procédures de développement rural (PAR - plans d'aménagement ruraux - chartes intercommunales, Leader....) en FrancheComté. À partir de 2000, responsable administratif d'un pays ou d'une intercommunalité dont la ville centre compte moins de 5000 habitants, ou personne considérée par le responsable comme "l'agent de développement ». La " date d'entrée » s'entend comme l'année durant laquelle l'individu remplit pour la première fois l'une des deux conditions ci-dessus. L'enquête a atteint l'exhaustivité pour la période récente et un faible nombre (une dizaine) de personnes évoquées dans l'enquête par reconstitution de réseaux n'ont pas été retrouvées.

L'analyse de l'activité a été menée pour les 70 agents encore en poste dans la région, rencontrés en face-à-face. La méthode d'entretien a reposé sur une verbalisation de l'activité générée par le commentaire de l'agenda. En effet, l'activité des agents de développement est très largement relationnelle et génératrice de réunions repérées dans les agendas. De ces entretiens, nous avons dégagé les difficultés récurrentes, pour repérer le "travail " au sens de Dejours entendu comme "confrontation à ce qui résiste " (1994), et les catégories mobilisées par les agents pour hiérarchiser l'activité et définir ce qu'est, selon eux, un véritable agent de développement.

Pour la reconstitution des parcours, nous avons collecté des éléments types que l'on retrouve dans un CV (curriculum vitae) : liste des postes occupés avec statut de l'organisme employeur, lieu d'emploi, statut de l'emploi et enfin domaines d'activité. Ce recueil de données a été complété, pour les 25 autres agents, par des entretiens téléphoniques.

\section{TROIS MOMENTS DU DÉVELOPPEMENT RURAL EN FRANCHE-COMTÉ}

Les modalités d'émergence et de stabilisation de la figure de l'agent de développement en milieu rural sont fortement déterminées par le contexte institutionnel de l'aménagement rural et de la coopération supra-communale (Jeannot, 2005). Les changements des institutions, liés aux deux phases de décentralisation en particulier, impulsent un mouvement que l'on retrouve, parfois avec un léger décalage temporel, dans le statut des agents, leurs profils ou dans les modalités locales de constitution de collectifs.

Trois moments du contexte institutionnel du développement rural peuvent être dégagés. Dans un premier temps, les démarches de développement autour des plans d'aménagement ruraux (PAR), après 1970, sont portées par les directions départementales 
de l'Agriculture auprès de communes non organisées. Quelques années après la décentralisation, vers 1985, cette aide centralisée disparaît ; les agents de développement demeurent uniquement là où des élus locaux ou des associations liées au milieu agricole portent un projet d'action à un niveau supracommunal. Enfin, après 1995 et de manière très rapide après 2000, les agents de développement sont employés par les nouvelles intercommunalités et pays créés par les lois qui instaurent de fortes incitations financières au regroupement ${ }^{3}$. Les PAR, et ensuite les projets portés après la décentralisation, vont consacrer l'échelle appelée alors des «petites régions » ou des pays. Si on compare la carte des PAR avec celles des actuels pays, on observe que les grandes lignes des découpages seront largement reprises dans l'application de la loi Voynet.

Les statuts des agents sont définis par ce cadre institutionnel. Le mouvement qui permet de passer d'une action centrée sur l'offre d'équipement à une démarche de développement autour des plans d'aménagement ruraux, après 1970, est porté par des ingénieurs fonctionnaires dans les directions départementales de l'Agriculture; le travail de proximité est accompli grâce au recrutement d'agents contractuels de l'État ou du département, soutenus, dans certains cas, par des agents des chambres d'agriculture. Après la décentralisation, cette aide centralisée disparaît progressivement; les élus locaux recrutent un agent qui doit «se construire son poste» en cumulant la participation à diverses procédures qui incluent des budgets «d'ingénierie ». À cette époque, les agents de développement sont, pour la plupart, employés sous CDD (contrat à durée déterminée) de droit privé dans des associations para-administratives. Enfin, les intercommunalités et pays doivent en principe recruter des agents statutaires de la fonction publique territoriale. Mais en pratique, cela n'est pas encore le cas ; parmi les trente deux agents recrutés à partir de 2000 , seuls quatre ont passé un concours ${ }^{4}$.

\footnotetext{
${ }^{3}$ Loi administration territoriale de la république, 6 février 1992 ; loi Pasqua, 4 février 1995 (LOADT) ; loi Voynet, 25 juin 1999 (LOADDT) ; loi Chevènement, 12 juillet 1999

${ }^{4}$ Parmi ceux entrés avant cette date, aucun n'avait passé un concours de la fonction publique territoriale à l'entrée, six ont été intégrés dans la fonction publique territoriale dont quatre sur concours et deux sur des vagues de régularisation des hors statuts.
}

Ces conditions statutaires ont également un impact sur les profils de formation des jeunes ${ }^{5}$ agents recrutés. Dans la première et la dernière période, les profils se répondent en partie par la concentration thématique (géographie et aménagement durant les années 70 , formations spécialisées en géographie et développement pour les plus récemment arrivés) et par le niveau de recrutement (ingénieur ou maîtrise, versus DESS - diplôme d'études supérieures spécialisées). L'atypicité des profils des recrutés de la période intermédiaire, 1980-1999, ressort d'autant mieux : flottement thématique dans les années 80 et 90 avec des profils très variés pour plus d'un tiers des entrants, flottement du niveau de recrutement avec une part importante d'agents ayant quitté leur formation initiale au niveau BTS (brevet de technicien supérieur) ou inférieur. Cela inclut en particulier des agents issus du milieu de l'animation ou de la formation pour adultes: bon nombre d'entre eux ont d'ailleurs été recrutés sur des postes financés par Jeunesse et Sport pour l'animation en milieu rural. Ils sont entrés entre 1980 et 1997. Cela inclut aussi des personnes ayant eu un parcours lié au militantisme agricole: dix agents ont été engagés activement, et souvent sur un mode militant, dans la recherche de formes alternatives au modèle dominant de développement agricole. Les agents recrutés à des bas niveaux de formation initiale, et qui vont faire preuve de capacité d'initiative importante, vont investir la formation continue diplômante pour faire reconnaître une capacité pratique d'action et faire valoir un engagement personnel fort. En effet, parmi les treize agents recrutés entre 1980 et 1999 qui ont un niveau III (niveau brevet de technicien supérieur) ou inférieur, huit ont mené à terme une formation continue diplômante, dont cinq à un niveau maîtrise ou DESS.

On observe la même temporalité s'agissant des formes de collectifs professionnels. À la première période correspondent des relations interpersonnelles fortes et durables mais peu organisées entre les agents pionniers; à la deuxième correspondent des tentatives d'organisation du milieu associant élus et agents de développement ; à la troisième période, des

\footnotetext{
${ }^{5}$ La moyenne d'âge d'entrée globale est de 28 ans et ceci de manière assez régulière au cours du temps.
} 
collectifs organisés directement par l'administration, dont l'action d'animation du milieu professionnel par le conseil général de Haute Saône offre la forme la plus développée.

\section{LA CONVERGENCE DES RÉFÉRENTIELS}

Les référentiels métier ont été produits par une partie seulement du groupe social des agents de développement. Dans le cas de la Franche-Comté, les profils des agents concernés illustrent cette spécificité. La première tentative est initiée par une des pionnières des plans d'aménagement ruraux. Elle travaille à la Direction départementale de l'Agriculture (DDA), et sera employée ensuite par une école d'agriculture. Elle réalisera quelques entretiens auprès d'agents de développement en vue d'animer le milieu local. La seconde tentative émane d'une responsable d'un bureau d'études qui va organiser des réunions locales des agents de développement et contribuer à un référentiel national (ARISTEE) ; en outre, le dirigeant de 1'association ADL 70, qui a connu une mobilité professionnelle entre plusieurs régions, participe activement à la rédaction du référentiel de l'association «Agents de développement, un métier à défendre ». Dans tous les cas, ces référentiels ont été portés par des agents combinant une certaine expérience et une certaine mobilité professionnelle. À la lecture du corpus rassemblé par la Plate-forme des métiers du développement, qui regroupe l'essentiel des associations de professionnels dans les domaines $\mathrm{du}$ développement urbain et rural, deux caractéristiques des référentiels métier produits sur les agents de développement s'imposent. En premier lieu, à une exception près, ces documents ne sont pas élaborés uniquement par les professionnels mais ont été coproduits avec les institutions en charge des politiques de développement. En second lieu, malgré des différences de présentation, ils ont une conception très homogène de ce que doit recouvrir un référentiel.

Malgré la diversité de leurs formes, ces référentiels se construisent autour de deux types de composantes (les fonctions à tenir et les qualités des individus) et de deux principes de différenciation (selon une divi- sion du travail et selon des niveaux de maîtrise des individus). Le métier d'agent de développement peut être saisi en bloc autour de ces caractéristiques ou des lignes de différenciation proposées.

Les principales fonctions évoquées sont: le diagnostic effectué sur un territoire; l'animation d'un territoire, entendue comme la capacité à rassembler des acteurs et à faire émerger des projets ; l'accompagnement, le montage et le suivi de projet; le suivi de procédures administratives et du financement.

Ces macro-fonctions sont ensuite déclinées en fonctions élémentaires qui y concourent. Ces fonctions peuvent être présentées comme les «missions» de l'agent de développement, comme des «activités» ou comme des «compétences», chaque fonction étant alors précédée de la formule «être capable de », ou comme une liste de "savoirs » et « savoirfaire ». L'ordre et la manière de présenter les fonctions de différents niveaux peuvent varier aussi notablement, certains référentiels comme ARISTEE partant de fonctions transversales (mobiliser, mettre en relation, accompagner, concevoir, rechercher, éduquer, former, informer, communiquer, négocier). Ces différenciations de présentation portent la marque du consultant de référence mobilisé. Elles peuvent s'accompagner d'introductions méthodologiques parfois sophistiquées. Dans certains cas, plusieurs niveaux de référentiels sont entrecroisés. Mais dans l'ensemble, ces manières d'aborder le sujet ne modifient ni le fait qu'un référentiel est d'abord une liste de fonctions, ni la liste des fonctions de référence.

Les qualités des personnes peuvent être présentées comme des « valeurs », des « savoir être » des « aptitudes requises » ou des « qualités personnelles ». Ces qualités peuvent être présentées en des termes très généraux (curiosité, persévérance, pragmatisme, dynamisme, diplomatie, polyvalence, ....) ou correspondre à une certaine manière de concevoir le développement (capacité de susciter la participation de tous les acteurs, aptitude d'évaluation et de prise de recul face aux tensions du contexte local, capacité à gérer un conflit sans éviter la confrontation, capacité à mettre en confiance, à travailler en équipe, ...). Elles sont plus différenciées que les listes fonctionnelles. 


\section{Encadré 3 \\ Les commanditaires des référentiels}

Les commanditaires des référentiels étudiés se répartissent autour de trois pôles : les associations professionnelles, les organes publics en charge des politiques de développement et les centres de formation. Le référentiel produit par l'association nationale "Agents de développement, un métier à défendre » est le seul à correspondre à une initiative complètement autonome d'une association professionnelle. Le caractère limite de ce cas se traduit aussi par la taille du document, qui tient sur deux pages. Trois référentiels (ARADEL, ARISTEE, et Plateforme des métiers) ont été pilotés par une association professionnelle, réalisés par un collectif de membres de ces associations, mais soutenus financièrement par un organisme public (Programme européen ADAPT - Initiative communautaire concernant l'adaptation des petites ou moyennes entreprises au marché unique) pour le référentiel ARISTEE, le conseil régional du Rhône pour le référentiel de l'ARADEL lassociation des professionnels du développement économique en Rhône Alpes) et la DATAR (délégation à l'Aménagement du Territoire et à l'Action régionale), la Caisse des dépôts et consignation, la Délégation interministérielle à la Ville et le ministère de l'Agriculture pour le référentiel de la Plateforme des métiers. Un référentiel a été commandé par un organisme public (Délégation interministérielle à la Ville) en charge de la promotion du développement et confié à un laboratoire universitaire $\left({ }^{*}\right)$. Un référentiel a été produit par un organisme employeur (Parcs naturels régionaux de France). Deux référentiels ont été commandités par un organisme de formation (Délégation lorraine du Centre national de la fonction publique territoriale, Chaire Unesco - United Nations Educational Scientific and Cultural Organisation - sur la formation des professionnels du développement durable). Notons ici l'absence des organismes qui pèsent sur la définition des cadres d'emploi dans la fonction publique territoriale, comme le Conseil supérieur de la fonction publique territoriale. Ces initiatives sont ainsi prises aux marges de la politique de gestion des ressources humaines, au sein des collectivités territoriales principales employeuses de ces agents après la seconde phase de décentralisation.

$\left.{ }^{*}\right)$ : celui-ci porte sur le métier de chef de projet dans la politique de la ville. Il ne s'agit que d'un exemple parmi de nombreux référentiels métier consacrés à ce secteur et une activité soutenue d'échanges entre professionnels organisés autour d'organismes comme " profession banlieue » ou comme l'inter-réseau du développement social urbain.

Certains référentiels introduisent, au sein du métier générique d'agent de développement des différenciations. Celles-ci suivent soit la division du travail soit le niveau de maîtrise de l'agent.

À la lecture de ces documents, il ressort une représentation passablement partagée de ce qui doit faire référence pour les agents de développement. Derrière des terminologies qui, d'un point de vue théorique, peuvent correspondre à des approches très différentes (des «valeurs» ou des «savoir être»; des «missions» ou des « compétences »), et avec des regroupements variables, ce qui est visé en pratique est fondamentalement la même chose. Cette unité des référentiels se révèle aussi par ce qui n'est pas évoqué. Ce qui rassemble ces référentiels est en effet un commun gommage des effets de contexte, gommage du contexte de l'organisation dans laquelle est affecté l'agent, qui conduit à négliger des dimensions importantes du travail et des compétences, gommage du contexte du marché du travail dans lequel il doit évoluer, qui amène à focaliser l'attention sur une forme de mobilité minoritaire dans le groupe étudié.

\section{DES RÉFÉRENTIELS EN DÉCALAGE PAR RAPPORT AU TRAVAIL ET AUX COMPÉTENCES}

La comparaison entre ces référentiels et les observations menées révèle une prise en compte limitée de certaines spécificités du travail, comme les relations avec les élus et les niveaux de prise d'initiatives. 


\section{Le travail avec les élus est sous-estimé}

Le premier décalage qui apparaît, lorsque l'on confronte la verbalisation de l'activité à ces référentiels, concerne un aspect important du travail. $\mathrm{Si}$ par travail on entend, en suivant Dejours, la « confrontation à ce qui résiste » (Dejours et Molinier, 1994), il ressort que la manière d'établir des relations avec les élus, qui est passablement euphémisée dans les référentiels, est au cœur du travail des agents de développement. Les personnes interrogées présentent en effet le bon équilibre de cette relation comme un des aspects les plus délicats de leur activité, et sa maitrise comme un des points importants de leur savoir-faire.

Le développement local vise à transformer la société, mais de fait l'action de transformation porte aussi sur les institutions qui l'encadrent. S'il fallait dresser à gros traits un bilan de ces trente années de développement local, on pourrait dire que ces agents, avant de contribuer à un développement économique, ont participé activement à un développement politique. Les élus sont la matière d'un travail, toujours masqué dans la manière officielle dont la profession parle d'elle-même, mais tout à fait essentiel pour l'activité quotidienne. Ceci se retrouve de manières diverses pour les trois périodes distinguées.

Pour la première période, l'image qui s'impose, en forçant le trait, est celle d'équipes commando qui débarquent sur un territoire et bousculent des ordres établis, en particulier en donnant la parole à des acteurs locaux non élus. Une première modalité de glissement d'une phase d'enquête et de discussion vers la mise en forme du politique local réside dans la constitution d'associations de développement pour fédérer de manière durable les plus motivés des participants aux commissions et passer à l'action. Après la phase de mobilisation par les réunions dans les villages, puis celle des réflexions dans les commissions, vient celle de la stabilisation du collectif dans une association. Il y a là un mélange de dirigisme technocratique et d'ouverture démocratique condensé dans l'expression : «créer des associations ». À côté des PAR en Haute Saône et dans le territoire de Belfort, les agents vont organiser des formations destinées aux élus sur des sujets comme les finances communales. Ces formations sont aussi ouvertes à des non-élus qui ont été sensibilisés à ces questions dans les commissions de travail des PAR. Plusieurs agents notent alors que ces non-élus constitueront le vivier de renouvellement du personnel politique local.

Avec la décentralisation, progressivement, les agents restent uniquement là où un élu assez volontariste fera appel à eux. La relation politique se joue alors largement dans le jeu des ajustements entre un agent et un élu et donc autant dans les parcours que dans le travail. La relation, lorsqu'elle existe, est présentée comme sans problème, en particulier sur la question du partage des rôles entre l'élu et l'agent de développement, partage considéré par les agents comme un arrangement de confiance entre individus. Si la relation, lorsqu'elle est établie, n'est pas l'objet d'une activité particulière, la préservation de la structure associative de développement l'est. L'élu et l'agent de développement partagent la même nécessité de légitimer cette intercommunalité informelle directement avec la population. Cela explique le caractère central, dans la plupart des référentiels, de la fonction de diagnostic territorial - qui sert autant à mobiliser la population et légitimer l'action de l'association qu'à définir l'offre. Ce n'est que dans les cas limites, lorsque l'élu est fragilisé, qu'il est remplacé à une élection ou que l'agent s'engage lui-même dans une carrière politique, que la relation est mise en cause, ce qui se traduit le plus souvent par le départ de l'agent de développement. Cela explique aussi l'importance de la notion de « projet » qui, contrairement aux fonctions de gestion, représente l'indépendance de l'agent de développement par rapport aux organisations des collectivités locales.

Lorsque l'on demande aux nouveaux arrivés, après la création des intercommunalités, en quoi ils se différencient de leurs aînés, c'est d'abord ce thème des relations au politique qu'ils évoquent. La volonté de retrouver des marques se retrouve même chez des anciens. Pour éviter ces travers, la position idéale décrite, dans un premier temps, par beaucoup, est celle du pur technicien qui se borne à présenter les options possibles en dressant un tableau neutre des avantages et inconvénients. Cependant, un tel détachement n'est pas tenable, l'agent ayant ses intérêts, sa culture professionnelle. Il s'agit alors de trouver un compromis entre la volonté d'apporter une touche 
personnelle, qui confère sens et intérêt au travail, et le respect des prérogatives des élus. La réalité quotidienne de la séparation des rôles ne suit pas nécessairement le principe acquis. Il faut procéder à un ajustement continuel et beaucoup d'agents nouvellement arrivés considèrent ce savoir-faire acquis sur le terrain comme un aspect des plus délicats.

La question des relations établies avec les élus, au centre des récits recueillis, n'est pas totalement absente des référentiels; plusieurs mettent en avant la subtilité nécessaire dans la relation: "avoir le sens de la relation aux élus, aux partenaires » (référentiel ARISTEE, p. 34). Le dernier référentiel en date (référentiel Plateforme des métiers) suggère ce thème d'entrée en soulignant, dans la première macro-compétence «Aide à la décision», que «Le développeur n'est pas un décideur», manière de recadrer les rôles. Cependant, la question est toujours abordée sous l'angle des prédispositions des individus, sans prendre en compte le poids du contexte institutionnel.

Les référentiels mettent en avant des fonctionnalités qui semblent neutres par rapport au politique, comme le diagnostic territorial ou le projet, et qui pourtant correspondent directement à un modèle particulier de la relation aux élus. Dans cette relation, l'agent coproduit avec l'élu une action qui se bâtit sa propre légitimité sur le territoire, s'inscrivant par là clairement dans une représentation de la seconde période. Mais le poids de la forme référentiel, cette volonté d'exprimer à travers des fonctions, ne permet pas complètement de désigner ce qui est visé et de caractériser la spécificité du travail que les promoteurs de ces référentiels cherchent à préserver.

\section{Le degré d'initiative des agents est occulté}

Si derrière le terme " compétence », on met principalement l'accent sur la prise d'initiative responsable, alors là encore cette dimension semble largement occultée par les référentiels, alors que l'enquête révèle que les agents mettent cette question au cour de leur définition de la spécificité du métier d'agent de développement. Philippe Zarifian (2002) définit la compétence à partir de l'initiative (capacité à initier l'action) et de la responsabilité (assumer les conséquences des initiatives), ces deux éléments étant complétés par une « intelligence pratique » qui permet de comprendre une situation singulière et pas seulement $d$ 'appliquer un savoir et une inscription dans un collectif qui seul autorise l'action efficace. Le commentaire d'agenda fait apparaître des différences notables à la fois dans les activités menées par des agents qui portent le même titre et dans leur niveau d'autonomie. En outre, les agents les plus anciens hiérarchisent ces pratiques selon l'ampleur de l'espace d'initiative, en distinguant une véritable pratique de développement d'une simple activité bureaucratique de gestion des dossiers associés aux thématiques du développement. Cette hiérarchie proposée par les agents peut être synthétisée dans le tableau 1.

Ici aussi, l'investissement des différents niveaux de pratique n'est pas lié aux seules caractéristiques des individus. Le contexte organisationnel favorise en effet plus ou moins la capacité d'initiative. Les mêmes agents les plus anciens déclarent d'ailleurs avoir vu, au cours du temps, leur marge d'initiative réduite par une nouvelle division du travail avec les élus et par l'accroissement des fonctions gestionnaires dans leur quotidien. L'activité qui consistait à aller au-devant d'interlocuteurs nouveaux parmi les entrepreneurs et la population, qui constituait une bonne part de l'originalité de leur fonction, est soit davantage contrôlée dans des cadres institués comme les conseils de développement ${ }^{6}$, soit a tout simplement disparu. Si on rapproche cette évolution de celle des qualifications scolaires à l'entrée, évoquée précédemment, on observe un curieux chassé-croisé entre une montée en qualification et une baisse de capacité d'initiative. En effet, les nouveaux recrutés, qui sont très contraints dans leur activité, sont pour la plupart titulaires d'un DESS alors que les agents recrutés juste après la décentralisation et qui disposaient d'une forte autonomie avaient pour beaucoup des niveaux de qualification bien plus faibles.

Cette différenciation des niveaux de pratique est encore moins saisie par les référentiels du corpus que la relation aux élus. L'approche fonctionnelle partagée par ces référentiels classe dans la même fonction ces différents niveaux de pratique; de plus,

\footnotetext{
${ }^{6}$ Organismes consultatif des pays qui peuvent être ouverts à la société civile.
} 
Tableau 1

Hiérarchie de niveaux de pratique établie par les agents de développement local

\begin{tabular}{|l|}
\hline Dossiers financiers : \\
\hline - Instruction \\
\hline - Recherche de nouvelles sources de financement \\
\hline - Ajustement des finances au projet \\
\hline Développement économique : \\
\hline - Suivi de procédures \\
\hline - Animation d'un collectif d'entrepreneurs \\
\hline Ouverture à la société civile : \\
\hline - Absence de contact hors élus \\
\hline - Organisation de réunions à la demande des élus \\
\hline - Recherche active d'interlocuteurs nouveaux \\
\hline
\end{tabular}

Source : Jeannot, 2006, : entretiens (commentaire d'agenda).

les principes de différenciation retenus selon des spécialisations ou des niveaux de maîtrise très généraux ne permettent pas de saisir de telles variations.

Les référentiels produits mettent en avant des qualités comme la diplomatie ou la capacité à mettre en confiance ou différencient de manière très générale le niveau de maîtrise plutôt que de clairement hiérarchiser des niveaux d'engagement dans l'activité directement observables. Or, d'une certaine manière, ce que les agents de cette génération cherchent à défendre, c'est justement une certaine capacité d'initiative. Le fait d'avoir utilisé la mise en avant du modèle d'un professionnel indépendant de l'organisation comme instrument de cette liberté empêche les agents de formuler la question de la capacité d'initiative au sein des organisations qui les emploient.

\section{UN DÉCALAGE PAR RAPPORT AUX PARCOURS PROFESSIONNELS}

Des décalages apparaissent aussi entre la représentation d'un métier d'agent de développement exercé au cours d'une carrière, en arrière-plan de ces référentiels, et la réalité des parcours des quatre vingt quinze agents ayant occupé, à un moment, un poste d'agent de développement rural en Franche-Comté.

Tout d'abord, en privilégiant les dimensions fonctionnelles, les référentiels ne permettent pas de mesurer l'importance accordée par les employeurs à la maîtrise thématique des dossiers. Tous les référentiels sont conçus de telle manière qu'il n'y ait pas de coupure entre les agents de développement en milieu rural et les chefs de projet qui sont leurs homologues dans le domaine des banlieues populaires. Or la reconstitution des parcours professionnels montre la forte étanchéité entre ces deux professions. Si on décompte le nombre d'agents de développement de la population étudiée qui ont, dans un poste antérieur ou postérieur, abordé un domaine particulier (par exemple qui ont travaillé dans le domaine de l'environnement) un constat s'impose : ces parcours hors des fonctions proprement dites d'agent de développement se concentrent autour d'un premier cercle limité d'activités qui, pour la plupart, correspondent à des thématiques que les individus ont rencontré ou vont rencontrer dans leur travail d'agent de développement rural ( $c f$. schéma 1). Ainsi, par exemple, un grand nombre d'agents de développement sont confrontés à des questions relatives au tourisme et un certain nombre d'entre eux passeront par les comités départementaux du tourisme. 
Schéma 1

\section{La mobilité fonctionnelle des agents de développement}

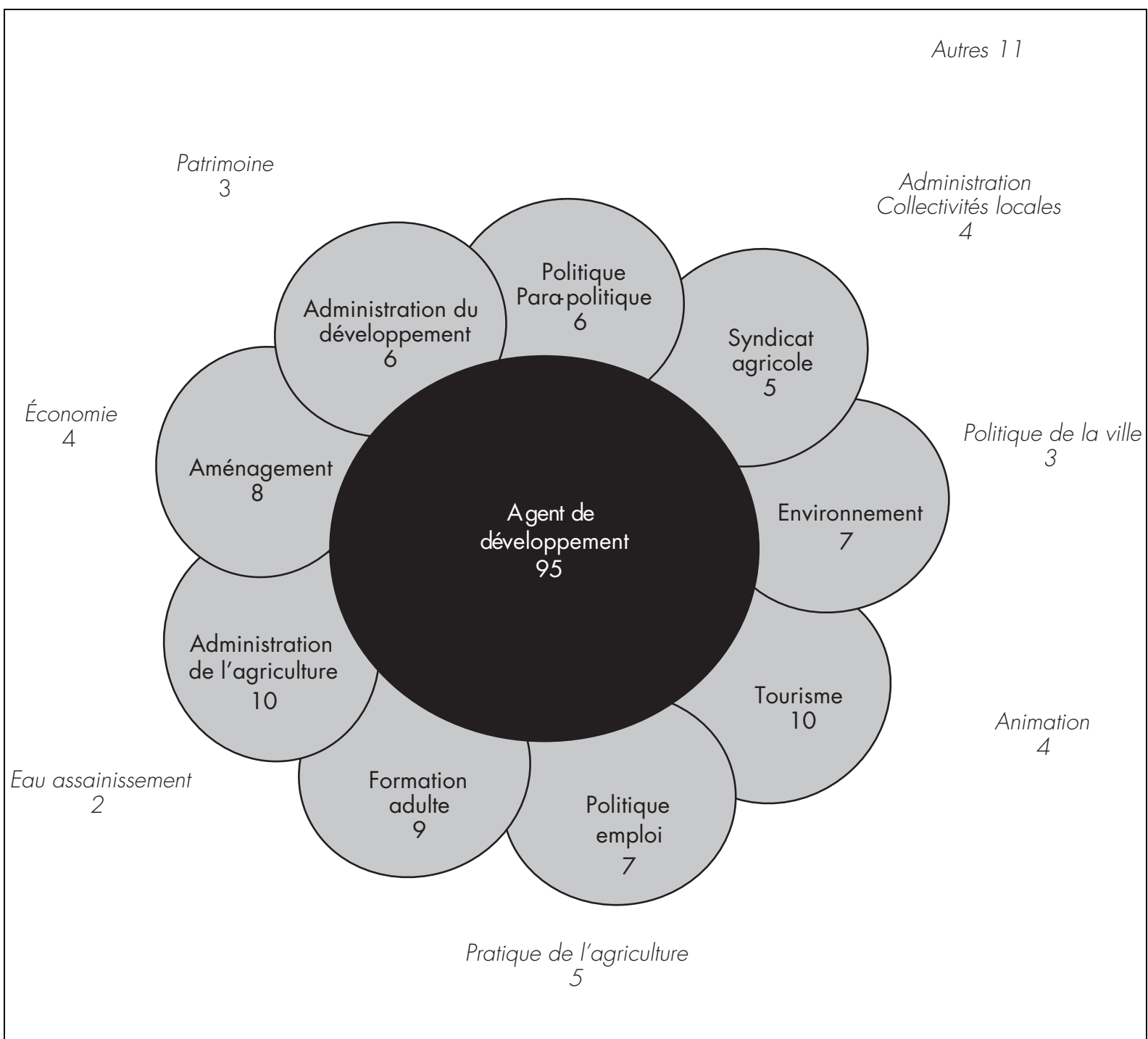

Lecture : parmi les 95 agents qui ont occupé un poste d'agent de développement, en Franche-Comté, 9 ont occupé un poste dans le domaine de la formation pour adultes et deux dans le domaine de l'eau-assainissement à un moment de leur carrière. Les positions dans les «pétales » correspondent à des thèmes abordés dans l'activité d'agent de développement, tandis que les positions « hors pétales » ne correspondent pas à cette activité.

Source : Auteur - reconstitution des parcours de 95 agents en Franche-Comté.

Le décalage apparaît ensuite par rapport à la mobilité géographique. Les référentiels se présentent comme des référentiels de "métier» et non comme des fiches de poste. Le «métier», comme le souligne Françoise Piotet (2002), suppose une certaine capacité à faire la même chose dans des situations proches. Et de fait, ces référentiels visent, de manière implicite, la figure d'un agent de développement «professionnel» mobile. Or les parcours d'agents ayant exercé plusieurs postes d'agent de développement dans des organisations différentes ou d'agents ayant travaillé dans des bureaux d'études sont minoritaires par rapport à ceux pour qui la fonction d'agent de développement n'a constitué qu'un moment de leur carrière ou encore de ceux qui n'ont pas connu de mobilité. 
Parmi les premiers arrivés, des agents contractuels de l'État, du département ou des chambres d'agriculture vont connaître une mobilité institutionnelle faible. Ils vont poursuivre une carrière relativement classique de fonctionnaire dans leur institution d'origine. S'y ajoutent des agents qui ont débuté leur carrière sur un statut précaire d'agent de développement pour la terminer dans les administrations de l'État, de la région ou du département. Cinq d'entre eux vont investir le domaine encore vierge des aides européennes, domaine qu'ils ont été les premiers à aborder dans leur action de développement local. Quinze agents ont des parcours à faible mobilité institutionnelle ; c'est le modèle dominant pour cette période.

Parmi les derniers arrivés, on observe des parcours qui se sont déroulés exclusivement dans les intercommunalités et pays. Les plus récemment arrivés, dont la carrière se réduit à un poste d'agent de développement d'une intercommunalité ou d'un pays, ont par construction une carrière qui relève de cette définition. S'y ajoutent des parcours d'agents plus anciens qui n'ont pas connu de mobilité géographique et sont passés d'agent de développement à secrétaire général de mairie ou d'intercommunalités. Quatorze agents entrés avant 2000 sont restés sur le même territoire depuis leur entrée dans le métier (en changeant éventuellement d'organisme) dont six entrés avant 1995.

Il est possible, par contraste, de rechercher des parcours marqués par une mobilité de type professionnel, en isolant les agents qui auraient occupé plusieurs postes d'agent de développement sur des territoires différents. Le premier constat remarquable est le faible nombre des agents qui ont occupé ne serait-ce que deux postes d'agent de développement sur des territoires différents. Neuf agents entrés avant 2000 relèvent de cette catégorie. Pour deux d'entre eux, ces postes, mêmes multiples, ne représentent qu'une petite part de leur parcours. Et deux sont entrés relativement récemment (en 1999), ce qui ne permet pas de juger du développement de la carrière. Il reste donc cinq agents qui correspondent à la figure d'un agent de développement mobile, enrichissant au fil des postes son expérience. À ces agents, on peut ajouter douze agents entrés avant 1999 et qui sont passés par un bureau d'études privé assurant des prestations dans le développement rural. Certains agents (cinq) qui se sont trouvés à nouveau sur le marché, après la décentralisation ou suite à des difficultés, ont créé leur propre poste afin de rester dans le même domaine lorsque les conditions sont devenues difficiles.

Cette faible mobilité au sein du métier d'agent de développement ne semble pas être une spécificité franc-comtoise. Une enquête menée par l'UNADEL $(1995)^{7}$, avant le grand développement des intercommunalités, permet de généraliser cette tendance. Si sur 556 agents de toutes les régions ayant répondu à l'enquête, 34,4\% déclarent ne pas occuper un premier poste d'agent de développement (ce qui semble supérieur au cas comtois), le développé des intitulés de ces postes révèle que 5,6\% ont été « agent de développement » et 2,7\% « animateur », expressions les plus classiques pour désigner cette fonction. De même, on retrouve la même absence de mobilité du développement social urbain vers le rural, seuls 2,3\% se reconnaissant dans l'appellation «chef de projet », appellation consacrée pour le développement en banlieues. Le modèle de mobilité semble plus être attaché au cadre institutionnel qu'au domaine abordé (mobilité au sein d'une administration d'État ou d'un réseau de chambres d'agriculture pour la première période, faible mobilité traditionnelle au sein des collectivités territoriales pour la dernière période), le passage par un bureau d'études semble à toutes les périodes un accélérateur de mobilité tant géographique que thématique. Selon plusieurs agents qui ont travaillé dans le rural et espéré déployer leur carrière en s'orientant vers l'urbain, l'étanchéité entre les secteurs serait due à la réticence des élus à recruter un agent ne maîtrisant pas les éléments du contexte institutionnel et juridique.

Pour la représentation de la mobilité, le fait que ces référentiels glissent d'une analyse du poste à celle d'un métier conduit à mettre en avant le modèle d'un parcours professionnel enchaînant les postes d'agent de développement, alors que ce type de parcours est

\footnotetext{
${ }^{7}$ La méthode qui repose sur la réponse volontaire à un questionnaire tend à sur-représenter les agents qui se reconnaissent dans un « métier » d'agent de développement et en particulier ceux qui auraient eu une mobilité entre plusieurs fonctions d'agent de développement. En outre, il est remarquable qu'alors que plusieurs questions étaient construites pour cerner la mobilité des agents, ces éléments ne sont pas repris dans les conclusions de l'UNADEL (Union nationale des acteurs et des structures du développement local).
} 
très minoritaire et que la mobilité géographique correspond à la pratique dominante des collectivités territoriales. De même, le mécanisme de mobilité par contiguité thématique, qui semble déterminé par la préoccupation de l'employeur de recruter quelqu'un qui maîtrise immédiatement les dossiers, en particulier dans leur dimension administrative, dessine des parcours cohérents mais qui s'inscrivent davantage dans la logique de carrières administratives bien gérées que dans le moule de la profession. Le fait en particulier que tous les chargés de mission "politiques européennes » des conseils généraux de la région soient d'anciens agents de développement illustre particulièrement ce type d'évolution.

Une telle représentation a certes pu correspondre en partie à la réalité de nombreux agents de développement durant la période située entre la décentralisation et la loi Chevènement; cependant, tant pour certains agents travaillant en bureau d'études pour les collectivités territoriales que pour d'autres ayant exercé les fonctions d'agent de développement dans des collectivités territoriales différentes, cette représentation apparait de plus en plus décalée par rapport à la situation des nouveaux agents recrutés après la généralisation des intercommunalités.

De tels décalages sur les parcours peuvent en partie relever d'une visée performative propre à l'exercice du référentiel, et en particulier indiquer aux employeurs des possibilités de recrutement élargies ; cependant, ils semblent aussi largement correspondre à une projection, par ceux qui sont au cœur de la rédaction des référentiels, de leurs propres parcours qui ne sont plus aujourd'hui représentatifs. La question de la pertinence de ce modèle de la profession est aujourd'hui directement posée comme un conflit de générations au sein des agents de développement. Les agents de développement comtois qui ont participé activement aux associations nationales et à la rédaction de ces référentiels métier correspondent aux premiers profils. Les nouveaux venus, aujourd'hui majoritaires, recrutés sur des statuts précaires de contractuels dans les nouvelles intercommunalités et pays, aspirent, pour leur part, à intégrer la fonction publique territoriale; ils attendent des aménagements aux concours de la fonction publique territoriale qui permettent, en particulier, de limiter le poids des épreuves de droit pour lesquels, ayant plutôt des profils de géographes, ils n'ont pas été préparés. Et s'ils partagent toujours les valeurs des fondateurs du développement rural et recherchent volontiers des occasions d'échange entre collègues, ils ne se reconnaissent qu'assez peu dans le projet de constitution d'une profession d'agent de développement.

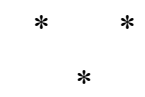

La mise en regard d'un corpus de référentiels et d'une enquête sur la pratique et les parcours des agents de développement permet de mettre en évidence de nombreux décalages. Trois sources à ces décalages apparaissent alors. Tout d'abord, des limites propres à tous les référentiels, comme la difficulté à traiter des problèmes du travail ou l'effort de décontexualisation destiné à ouvrir des possibilités nouvelles de mobilité. Ensuite, le fait que ces référentiels projettent une représentation de la position de ces agents inspirée de la figure du professionnel libéral relativement mobile et indépendant, qui ne correspond qu'aux agents qui justement rédigent ces référentiels. Mais le plus remarquable est que les moyens choisis, la mise en avant d'un modèle de profession d'une part et la rédaction d'un référentiel d'autre part, ne permettent pas vraiment au projet de ces rédacteurs de préserver une certaine conception du développement ouverte à la population et qu'ils pourraient partager avec les nouveaux venus.

Ceci ne signifie pas que les deux questions posées d'emblée, le maintien de l'originalité des interventions du développement local et la protection des agents, ne soient plus pertinentes; mais d'autres formes que le modèle de l'autonomisation et de la reconnaissance institutionnelle d'une profession d'agent de développement doivent être explorées en prenant mieux en compte la situation nouvelle créée par le fort développement des pays et des intercommunalités. Cela pourrait conduire à prendre acte de la volonté des nouveaux venus d'entrer dans la fonction publique territoriale, soit en pesant collectivement sur les instances qui définissent les conditions d'accès pour que des concours n'intégrant pas d'épreuves de droit soient créés, soit en faisant évoluer les formations existantes pour qu'elles préparent aux concours actuels. On pourrait intégrer, dans la lignée de 
l'expérience du conseil général de la Haute Saône, des actions administratives facilitant l'échange professionnel au niveau local et la mobilité à cette échelle. Enfin, pour promouvoir une certaine conception du développement incluant en particulier une ouverture à la société civile, on pourrait renouer avec des organisations qui, comme les premières obser- vées en Franche-Comté, allaient au-delà du cercle professionnel. Des exercices d'analyse de la pratique ne seraient pas forcément à exclure. La réduction effective de prise d'initiative des agents de développement local et l'explicitation des conditions qui la génère pourraient constituer des arguments aptes à alimenter le débat.

\section{Bibliographie}

Brévan C., Picard P. (2000), Une nouvelle ambition pour les villes, de nouvelles frontières pour les métiers, Rapport à Monsieur Claude Bartolone, Ministre délégué à la Ville.

Combes M.-C. (2002), « La compétence relationnelle : une question d'organisation », Travail et Emploi, $\mathrm{n}^{\circ}$ 92, octobre, pp. 5-18.

Dadoy M., Henry Cl., Hillau B., De terssac G., Troussier J.-F., Weill-Fassina A. (dir.) (1990), Les analyses du travail enjeux et formes, Collection des études du Céreq, $\mathrm{n}^{\circ}$ 54, $240 \mathrm{p}$.

Dejours Ch., Molinier P. (1994), « Le travail comme énigme », Sociologie du travail, hors série, pp. 3544.

Demazière D. (2004), "Médiation et médiateurs sociaux : entre nomination et professionnalisation », Formation Emploi, nº 86, pp. 11-23.

Dubar C., Tripier P. (1998), Sociologie des professions, Paris, Armand Colin, 256 p.

Dugué E., Malglaive G. (1990), Les employés face au changement, Paris, CNAM, C2F, p. 272.

Jeannot G. (2005), « Les métiers flous du développement local », Sociologie du travail, 47, 1, pp. 17-35.
Labruyère C. (1999), «La professionnalisation des emplois de servic », in Heurgon E., Stathopoulos N., Les métiers de la ville, Éditions de l'Aube, pp. 320328.

Oiry E., Sulzer E. (2005), « Le référentiel de compétence, entre outil scientifique et compromis social », in Jouvenot C., Parlier M., Élaborer des référentiels de compétences, ANACT, pp. 228-247.

Paradeise C. (1986), «Les marchés du travail fermés", in Le marché du travail, Cahiers $d u$ GRECO 041 .

Piotet F. (2002) (Éd.), La révolution des métiers, Paris, PUF.

Sirinelli J.-F. (1988), Générations intellectuelles: khâgneux et normaliens dans l'entre-deux guerres, Paris, Fayard.

Zarifian P. (2002), La politique de la compétence et l'appel aux connaissances à partir de la stratégie d'entreprise post-fordiste, Contribution au colloque de Nantes, 13 décembre 2002. 


\title{
Résumé
}

\section{À quoi ne se réfèrent pas les référentiels métier des agents de développement local ?}

\author{
Gilles Jeannot
}

Les référentiels métier consacrés aux agents de développement local en milieu rural s'avèrent décalés par rapport à la réalité du travail, des compétences ou des parcours de ces professionnels, révélée par une enquête exhaustive menée en 2003 en Franche-Comté. Ces décalages trouvent notamment leur origine dans l'a priori de décontextualisation partagé par ces référentiels. L'article s'interroge sur les effets pratiques de ces décalages entre la référence proposée et la réalité. II conclut que ces décalages sont contreproductifs par rapport à la visée de professionnalisation qui constitue la raison d'être de ces référentiels.

\section{Mots clés}

Référentiel métier, analyse du travail, agent de développement

Journal of Economic Literature : J 24 - Human Capital; Skills; Occupational Choice; Labor 


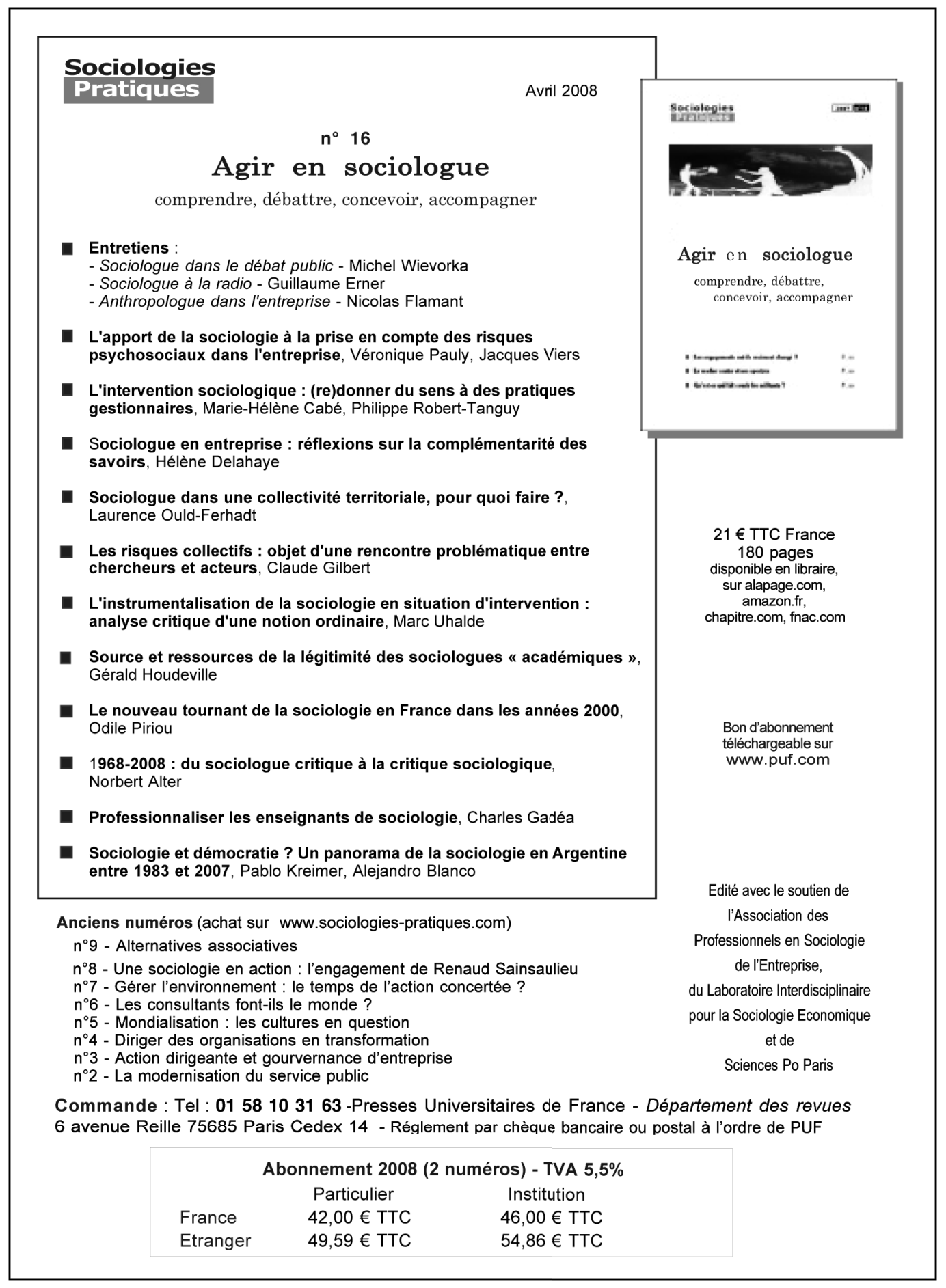

